\title{
Identification of a kidney-specific mouse organic cation transporter like-1 (mOCTL1)
}

\author{
Woon Kyu Lee ${ }^{1}$, Ji-Sun Hwang ${ }^{2}$, Cheol-Heui Yun ${ }^{3}$ \\ and Seok Ho Cha ${ }^{4,5}$ \\ ${ }^{1}$ Department of Laboratory Animal Medicine \\ Medical Research Center, College of Medicine \\ Yonsei University \\ Seoul 120-752, Korea \\ ${ }^{2}$ Department of Physiology and Biophysics \\ College of Medicine, Inha University \\ Incheon 400-712, Korea \\ ${ }^{3}$ Laboratory of Protein Engineering and Comparative Immunology \\ School of Agricultural Biotechnology \\ Seoul National University \\ Seoul 151-921, Korea \\ ${ }^{4}$ Department of Pharmacology and Toxicology \\ Center for Advanced Medical Education by BK21 Project \\ College of Medicine, Inha University \\ Incheon 400-712, Korea \\ ${ }^{5}$ Corresponding author: Tel, 82-32-890-0957; \\ Fax, 82-32-890-0957; E-mail, shcha@inha.ac.kr
}

Accepted 5 November 2007

Abbreviations: CCD, cortical collecting duct; DCT, distal convoluted tubule; EST, expressed sequence tag; G, glomerulus; OAT, organic anion transporter; OCT, organic cation transporter; OCTL, organic cation transporter like; РCT, proximal convoluted tubule

\begin{abstract}
Organic ion transporters are expressed in various tissues that transport endogenous and exogenous compounds including their metabolites. There are organic anion transporter (OAT), organic cation transporter (OCT), organic anion transporter like protein (OATLP) and organic cation transporter like (OCTL). Considering the variety of charged organic ionic compounds, the existence of numerous isoforms of organic ion transporters can be assumed. In the present study, we have searched for a new isoform in the expressed sequence tag (EST) database using human organic anion transporter 4 (hOAT4) amino acid sequence as a "query". We found a candidate clone (BC021449) from the mouse kidney cDNA library. This clone was identified as an ortholog of ORCTL3 or OCTL-1. The MOCTL1 cDNA consists of 2016 base pairs encoding 551 amino acid residues with 12 putative transmembrane do-
\end{abstract}

mains. The deduced amino acid sequence of mOCTL1 showed 35 to $40 \%$ identity to those of the other members of the OATs and OCTs. According to the tissue distribution, examined by Northern blot analysis, about a 2.4-kb transcript of mOCTL1 was observed in the kidney. About a 90-kDa band was detected when Western blot analysis in the mouse kidney was done by using antibody against synthesized oligopeptide of mOCTL1. The immunohistochemical result showed that mOCTL1 was stained at the glomerulus (the parietal epithelial cells and podocytes), pars recta of proximal tubule, distal convoluted tubules, connecting tubules and collecting tubules. From these results, we conclude that MOCTL1 may be a candidate for an organic ion transporter isoform in the mouse kidney.

Keywords: kidney; immunohistochemistry; organic anion transporters; organic cation transport proteins

\section{Introduction}

A great number of charged soluble molecules are eliminated through transporters in the kidney. They belong to solute carrier family (SLC) 22 family proteins. This transporter family includes the organic anion transporters (OATs), organic cation transporters (OCTs), zwiterion transporters (OCTNs), and several related genes (USTs and URAT1/RST) in mammals (Sekine et al., 2000; Eraly et al., 2004).

The mechanisms of cellular transport, involved in proximal tubular secretion of organic anions and cations, have been investigated in detail and are reviewed elsewhere (Pritchard and Miller, 1992; Roch-Ramel et al., 1992; Roch-Ramel and Diezi, 1997; Koepsell, 1998). The $p$-aminohippurate (PAH) and tetraethylammonium are well known as prototypical substrates of organic anion transporter and organic cation transporter, respectively. Common structural components for substrates of these transporters are a hydrophobic moiety and the existence of ionic or partial electrical charges (Ullrich, 1994, 1997). The six OATs (Sekine et al., 2000; Monte et al., 2004; Youngblood and Sweet, 2004), three OCTs (Grundemann et al., 1994; Okuda et al., 1996; Kekuda et al., 1999), three OCTNs (Tamai et al., 1997; Wu et al., 1998; Enomoto et al., 2002a) and a URAT1/RST (Enomoto et al., 2002b; Hosoyamada et al., 2004) contribute to 
movement of electrically charged or zwiterionic compounds between cells and their milieu.

Many lipid-soluble compounds are biotransformed to their hydrophilic metabolites, such as organic anions or organic cations. These charged compounds are difficult to move through the plasma membrane. The organic ion transporters play important roles in moving a numerous charged compounds and have multispecificity to the substrates. In addition, considering the numerous kinds of organic ionic substance in the body, it is likely that there are not yet identified isoforms of organic ion transporter.

In this regard, we have tried to discover new isoforms of organic ion transporter by performing homology screening. We searched the expressed sequence tag (EST) data based on employing the query "hOAT4". We here report the molecular cloning of a cDNA encoding a mouse organic cation transporter like protein, and its complete primary structure, tissue distribution, and intrarenal localization.

\section{Materials and Methods}

\section{Materials}

The materials used in the present study were purchased from following sources: The $\left[{ }^{32} \mathrm{P}\right] \mathrm{dCTP}$ from Amersham Pharmacia Biotech (Uppsala, Sweden), Taq DNA polymerase from Super-Bio Co., Ltd. (Suwon, Korea), TRI Reagent for RNA isolation from Sigma-Aldrich (St. Louis, MO), PCR primers, dNTP mixture and AMV reverse transcriptase XL from TaKaRa Korea Biomedical Co. (Seoul, Korea). All other chemicals utilized in this study were the highest purity available from commercial sources.

\section{RT-PCR and Isolation of mOCTL1}

EST database was searched for the query "hOAT4", and an EST clone, BC021449 was identified. Primers were designed based on the nucleotide sequence as following: forward primer, 5'-TTGCACAGGTCATGGCTGAAGTGG-3' and reverse primer, 5'-AAGTAGAGCTCAAAGCTGGACAC-3'. Using this set of primers, we performed RT-PCR on total RNA from the mouse kidney. The protocol for PCR was as follows: $94^{\circ} \mathrm{C}$ for $10 \mathrm{~s}, 57^{\circ} \mathrm{C}$ for 30 s, $72^{\circ} \mathrm{C}$ for $30 \mathrm{~s}, 35$ cycles (Kwak et al., 2005). The PCR product was subcloned into TA cloning vector and its sequence was confirmed. The $\left[{ }^{32} \mathrm{P}\right] \mathrm{dCTP}$ labeled probe was synthesized from the PCR clone and used for the screening of a mouse kidney cDNA library. A non-directional cDNA library was prepared from mouse kidney poly $(A)^{+}$RNA using the Superscript Choice system (Life Technologies), and the cDNAs were ligated into ZipLox EcoRI arms. Replicated filters of a phage library were hybridized overnight at $37^{\circ} \mathrm{C}$ in a hybridization solution $(50 \%$ formamide, $5 \times \mathrm{SSC}, 3 \times$ Denhardt's solution, $0.2 \%$ SDS, $10 \%$ dextran sulfate, $0.2 \mathrm{mg} /$ $\mathrm{ml}$ denatured salmon sperm DNA, $2.5 \mathrm{mM}$ sodium pyrophosphate, $25 \mathrm{mM}$ MES, and $0.01 \%$ Antifoam $\mathrm{B}, \mathrm{pH} 6.5)$, and washed at $37^{\circ} \mathrm{C}$ in $0.1 \times \mathrm{SSC}$ and $0.1 \%$ SDS (Kusuhara et al., 1999; Eun et al., 2001).

\section{Sequence determination}

Specially synthesized oligonucleotide primers were used for the sequencing of the mOCTL 1 cDNA by dye-termination method using $\mathrm{ABI}$ Prism ${ }^{\mathrm{TM}} 3730$ (CoreBio System Co., Ltd, Seoul, Korea).

\section{Computational analysis}

Peptide sequences of OAT family were obtained from GenBank (http://www.ncbi.nlm.nih.gov) and were aligned with CLUSTALW (http://clustalw.ddbj. nig.ac.jp/top-e.html). Dendrograms were generated from the CLUSTALW output using Tree-View (http: //taxonomy.zoology.gla.ac.uk/rod/treeview.html). Other sequence analysis was performed with DNAsis (Hitachi, Japan).

\section{Northern blot analysis}

A commercially available hybridization blot containing $\operatorname{poly}(A)^{+}$RNA from various mouse tissues [mouse 8-lane multiple tissue Northern (MTN) blot, Clontech] was used for the Northern blot analysis for mOCTL1. We used a full size of mOCTL1 cDNA as a probe. The master blot filter was hybridized with the probe overnight at $42^{\circ} \mathrm{C}$ according to the manufacturer's instruction. The filter was washed finally in a high stringency condition $[0.1 \times$ SSC $(1 \times$ SSC $=0.15 \mathrm{M} \mathrm{NaCl}$ and $0.015 \mathrm{M}$ sodium citrate) and $0.1 \% \operatorname{SDS}$ at $\left.65^{\circ} \mathrm{C}\right]$.

\section{Western blotting and test for antibody specificity}

Western blot analysis was performed following previous report with slight modification (Jung et al., 2005). For preparation of polyclonal antibody to mOCTL1, the rabbits were immunized with a keyhole limpet hemocyanin-conjugated synthesized peptide, CETRGQILKDTLQDLEQ corresponding to cysteine and the 17 amino acids of near the $\mathrm{COOH}$ terminus of mOCTL1.

The kidney from mouse (ICR) was perfused with 
ice-cold PBS (pH 7.4). Subsequently, kidney was removed and homogenized in 9 volume of lysis buffer containing $2.1 \mathrm{M}$ sucrose, $200 \mathrm{mM}$ HEPES (pH 7.4), $1 \mathrm{mM}$ EDTA and a protease inhibitor mixture $(1 \mathrm{mM}$ DTT, $1 \mathrm{mM}$ PMSF, $10 \mu \mathrm{g} / \mathrm{ml}$ leupeptin, $10 \mu \mathrm{g} / \mathrm{ml}$ trypsin inhibitor, and $2 \mu \mathrm{g} / \mathrm{ml}$ aprotinin) with Teflon/glass tissue homogenizer. After centrifugation at $500 \times \mathrm{g}$ for $5 \mathrm{~min}$, the supernatant was collected and centrifuged at 8,000 $\times g$ for $10 \mathrm{~min}$. The supernatants were collected and then centrifuged again at $100,000 \times g$ for $1 \mathrm{~h}$, and the pellets containing crude membrane fractions were used for Western blotting. Protein concentration of the prepared sample was determined by using BCA protein assay kit (Pierce, Rockford, IL). Equal amount of proteins were mixed with 2-fold concentrated loading buffer (100 $\mathrm{mM}$ Tris $\mathrm{HCl}, \mathrm{pH} 6.8,2.5 \%$ 2-mercaptoethanol, $10 \%$ glycerol, $4 \%$ SDS, $0.2 \%$ bromophenol and $200 \mathrm{mM}$ DTT), heated for $10 \mathrm{~min}$ at $100^{\circ} \mathrm{C}$ and then subjected to electrophoresis on 9\% SDSpolyacrylamide gels. Separated protein was transferred onto PVDF membrane (Millipore, Bedford, MA) by electro-blotting and non-specific binding sites were blocked by incubating with $5 \%$ non-fat dried milk in the TBST buffer [10 $\mathrm{mM}$ Tris $\mathrm{HCl}, \mathrm{pH}$ 7.4, $100 \mathrm{mM} \mathrm{NaCl}$ and $0.1 \%(\mathrm{v} / \mathrm{v})$ Tween 20] for 1 $\mathrm{h}$ with gentle shaking. The PVDF membrane was then incubated for $1 \mathrm{~h}$ at room temperature with the anti-mOCTL1 antibody $(1,000: 1)$. For absorption experiment, anti-mOCTL1 antibody was pretreated with synthesized oligopeptide antigen (200 $\mu \mathrm{g} / \mathrm{ml}$ ) for $1 \mathrm{~h}$ at $37^{\circ} \mathrm{C}$. Afterwards, membrane was incubated further for $1 \mathrm{~h}$ at room temperature with HRP-conjugated secondary antibody (1:3,800 dilution). After washing the membranes with TBST buffer, protein bands on X-ray film were visualized by using the ECL detection kit as described by manufacturer's instruction (Amersham Pharmacia Biotech, Buckinghamshire, UK).

\section{Immunohistochemical analysis}

For immunohistochemical analysis, the $1-\mu m$-thick vibratome sections were processed for immunohistochemistry using an indirect preembedding immunoperoxidase method. The sections were washed three times for 15 min each in PBS containing $50 \mathrm{mM} \mathrm{NH}_{4} \mathrm{Cl}$. They were then incubated for $4 \mathrm{~h}$ in PBS containing $1 \%$ BSA, $0.05 \%$ saponin, and $0.2 \%$ gelatin (solution $A$ ). The tissue sections were incubated overnight at $4^{\circ} \mathrm{C}$ with mOCTL1 antibody diluted 1:400 in 1\% BSA in PBS (solution B). After several washes with PBS containing $0.1 \%$ BSA, $0.05 \%$ saponin, and $0.2 \%$ gelatin (solution $\mathrm{C})$, the tissue sections were incubated for $2 \mathrm{~h}$ in a
1:100 dilution of a peroxidase-conjugated goat anti-rabbit IgG Fab fragment (Jackson ImmunoResearch Laboratories) in solution B. The tissues were then rinsed, first in solution $\mathrm{C}$ and subsequently in $50 \mathrm{mM}$ Tris- $\mathrm{HCl}(\mathrm{pH} \mathrm{7.6)}$. For the detection of HRP, the sections were incubated in $0.1 \%$ 3,3'-diaminobenzidine substrate in the Tris buffer for $5 \mathrm{~min}$. Then colorization of reaction was achieved by an addition of $0.01 \% \mathrm{H}_{2} \mathrm{O}_{2}$ for $10 \mathrm{~min}$. After being washed in the Tris buffer, the sections were dehydrated in a graded series of ethanol and embedded in Epon 812 (Polysciences, Warrington, CA).

\section{Results}

\section{Structural features of novel mOCTL1}

We have searched EST database and identified an EST clone, BC021449, and this clone showed identity to the mOATs and mOCTs. With using BC021449 as a probe, a positive clone was isolated by screening 320,000 plaques from the mouse kidney cDNA library. The clone was turned out to be a membrane protein (mOCTL1) consisting 2016 base pairs that encode for 551 amino acid residues. When the membrane topology was analyzed by using a software, TMpred, and performing Kyte and Doolittle hydrophobicity analysis, mOCTL1 showed 12 putative transmembrane domains (Figure $1 \mathrm{~A}$ and $1 \mathrm{~B}$ ). Both the $\mathrm{N}$-terminus and $C$-terminus were located in the intracellular space. As shown in Figure 1A, mOCTL1 contained four $\mathrm{N}$-glycosylation sites in the extracelluar surface between the first and second membrane spanning domains. mOCTL1 also contained six PKC phosphorylation sites in the intracellular surface.

\section{Relationship of mOCTL1 to other OAT and OCT family members}

We compared the mOCTL1 sequence to those of other members of the OAT and OCT family (Table 1). The amino acid homologies of OATs (including URAT1/RST) and OCTs were in the range of approximately $35 \%$ to $40 \%$. We aligned the peptide sequences of the known OATs, OCTs, OCTLs and mOCTL1 to generate a dendrogram delineating their phylogeny (Figure 2). The phylogenetic tree showed that mOCTL1 may be categorized to the OCT family.

\section{Tissue distribution of mOCTL1}

In order to demonstrate the tissue distribution of 
A

MAQFAQVMAE VGDFGRQVR LTILMGIPNF LAAFFIFGQV FMVLDEAHHC SVSWVKNHTF NLSAAEQLAI 70

$\underset{*}{\operatorname{SIPNDTTGRP}}$ ESCLMFRPPP DSASLEDILS HRFNETQACD SGWDYPENRP QSLKKEFDLV CDRKNLKKTS 140

3

4

QSVFMAGLLV GALVFGPVCD WIGRRPSLLM QVLLSGITSM ATAFVSSFEL YLALRFVLAT ANAGFLLSTN $\overline{\text { 210 }}$

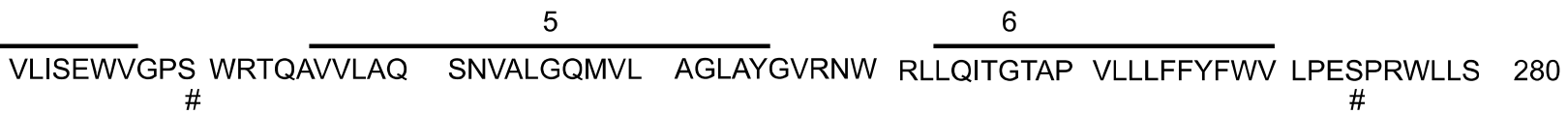

$\frac{7}{\text { VTLILIAV WFVDSLVYYS }}$

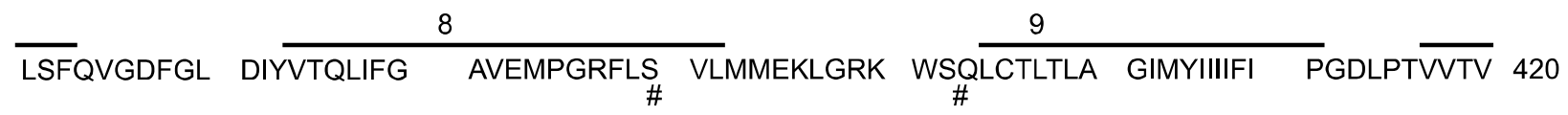

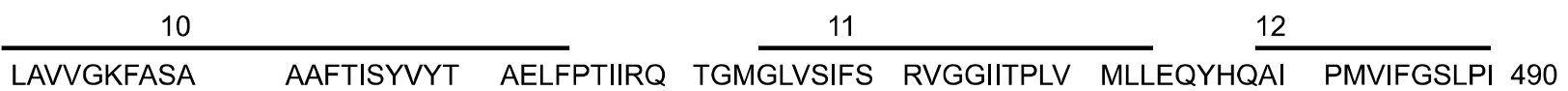

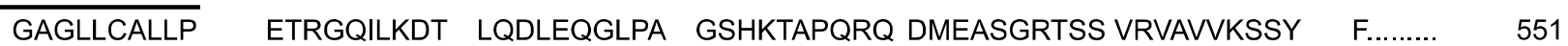

\section{B}

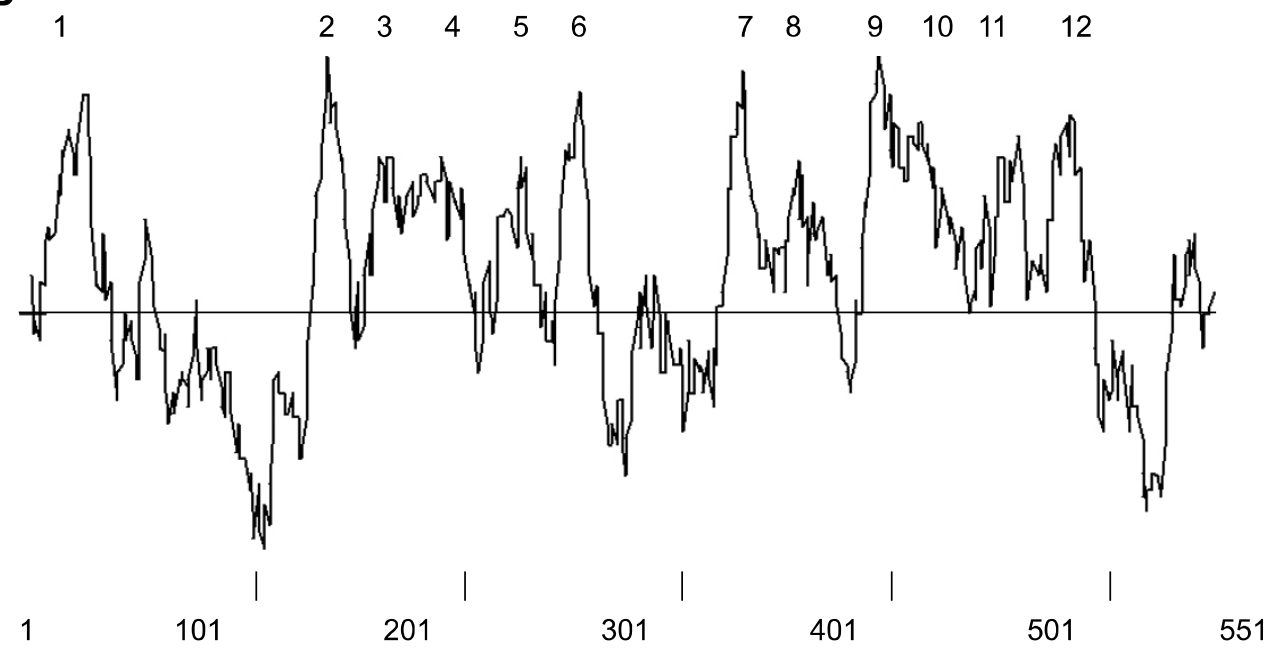

Figure 1. Amino acid sequence of mOCTL1 and hydropathy analysis. A. Amino acid sequence of mOCTL1. Potential $\mathrm{N}$-glycosylation sites are indicated by asterisks and PKC phosphorylation sites by sharps. B. Kyte-Doolittle hydropathy analysis of mOCTL1. Predicted membrane-spanning regions of mOCTL1 are numbered 1-12.

mOCTL1 mRNA, Northern blot analysis was employed for the tissues of various mouse organs (heart, brain, spleen, lung, liver, skeletal muscle, kidney and testis). When the hybridization was performed with the full length of mOCTL1, about a $2.4 \mathrm{~kb}$ transcript of mOCTL1 was observed in kidney at $12 \mathrm{~h}$ exposure (Figure 3 ). The expression of mOCTL1 transcripts was not detected in the 
Table 1. Percentage of amino acid identity among fully sequenced organic ion transporters.

\begin{tabular}{|c|c|c|c|c|c|c|c|c|c|c|c|c|}
\hline & mOAT2 & mOAT3 & hOAT4 & mOAT5 & mOAT6 & mOCT1 & mOCT2 & mOCT3 & hORCTL3 & hORCTL4 & \multicolumn{2}{|c|}{ RST/URAT1 mOCTL1 } \\
\hline mOAT1 & 40 & 50 & 42 & 40 & 48 & 38 & 37 & 36 & 38 & 33 & 46 & 39 \\
\hline mOAT2 & & 40 & 38 & 36 & 40 & 36 & 38 & 37 & 39 & 35 & 40 & 38 \\
\hline mOAT3 & & & 43 & 39 & 48 & 37 & 36 & 36 & 37 & 34 & 45 & 39 \\
\hline hOAT4 & & & & 45 & 45 & 35 & 34 & 36 & 38 & 34 & 51 & 38 \\
\hline mOAT5 & & & & & 40 & 34 & 35 & 35 & 38 & 33 & 45 & 36 \\
\hline mOAT6 & & & & & & 39 & 39 & 37 & 39 & 33 & 45 & 40 \\
\hline mOCT1 & & & & & & & 70 & 48 & 39 & 31 & 36 & 37 \\
\hline mOCT2 & & & & & & & & 50 & 37 & 32 & 35 & 35 \\
\hline mOCT3 & & & & & & & & & 36 & 32 & 35 & 36 \\
\hline hORCTL3 & & & & & & & & & & 38 & 39 & 73 \\
\hline hORCTL4 & & & & & & & & & & & 31 & 37 \\
\hline RST/URA & & & & & & & & & & & & 37 \\
\hline
\end{tabular}

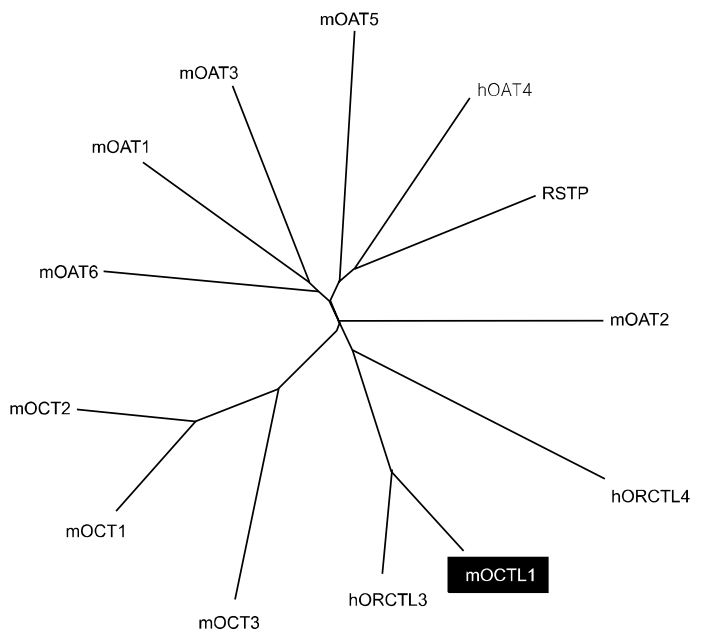

0.1

Figure 2. Dendrogram of the OAT and OCT family. Sequences of the indicated OAT and OCT family members were aligned with CLUSTALW and the alignment output was used to generate a dendrogram.

other tissues by even a long exposure (more than three days).

\section{Western blot analysis of mOCTL1 on mouse kidney}

Western blot analysis was performed on the membrane fraction prepared from mouse kidney. About $90-\mathrm{kDa}$ band was detected using antibody against mOCTL1. As shown in Figure 4, this immunoreactive band disappeared by using the pre-absorbed antibody with an oligopeptide antigen $(200 \mu \mathrm{g} / \mathrm{ml})$. Therefore we conclude that the mOCT1 is specifically present in the mouse kidney.

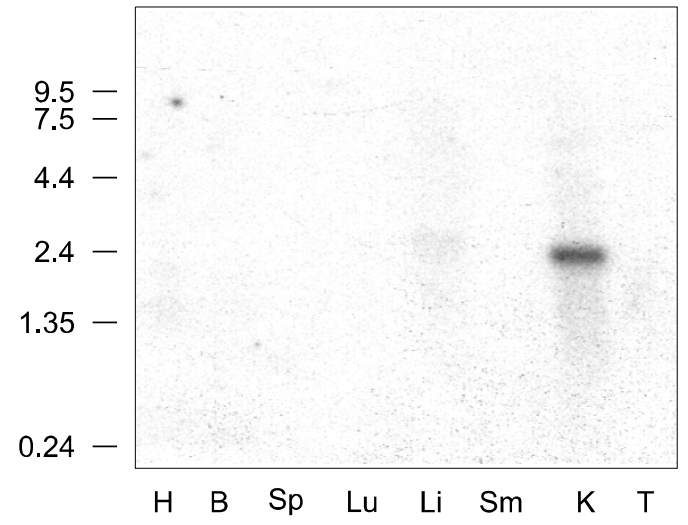

Figure 3. Northern blot analysis of mOCTL1. Mouse multiple tissue blot that contains $2 \mu \mathrm{g}$ of poly $(A)^{+}$RNA from 8 mouse tissues in each lane was probed with a ${ }^{32} P$-labeled coding region of mOCTL1 CDNA and was washed in a high stringent condition. $\mathrm{H}$, heart; B, brain; Sp, spleen; Lu, lung; Li, liver; Sm, skeletal muscle; K, kidney; T, testis.

\section{Immunohistochemistry of mOCTL1 in mouse kidney}

Light microscopic test on $1-\mu \mathrm{m}$ thick vibratome sections of mouse kidney demonstrated that there was a specific immunostaining of mOCTL 1 in the glomerulus (the parietal epithelial cells and podocytes) (Figure $5 \mathrm{~A}$ ). In proximal convoluted tubules and distal convoluted tubules, the immuno-reactivity was observed at the apical site (Figure 5B and C). In contrast, the immuno-staining to antibody in connecting tubules and collecting tubules revealed diffused pattern in cytoplasm (Figure 5C and D).

\section{Discussion}

In the present study, we report the isolation of mouse organic cation transporter like 1 (mOCTL1) from mouse kidney cDNA library and revealed its 


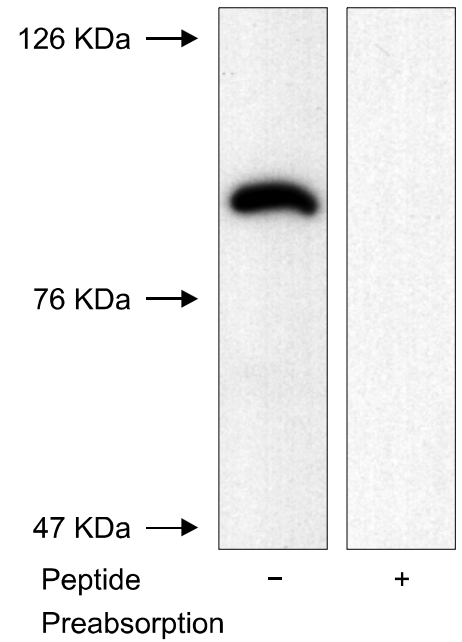

Figure 4. Western blot analysis of mOCTL1. Membrane fraction $(20$ $\mu \mathrm{g} /$ lane) was boiled with the same volume of $2 \times$ loading buffer for 10 min and separated by electrophoresis on 9\% SDS/PAGE gel. Protein transferred membrane was treated with the primary antibody against mOCTL1 (A) or pre-absorbed primary antibody (B). Afterwards, membrane was incubated for $1 \mathrm{~h}$ at room temperature with HRP-conjugated secondary antibody (1:3,800 dilution). After washing the membranes with TBST buffer, protein bands on X-ray film were visualized by using the ECL detection kit as described in the Materials and Methods. physical location. mOCTL1 cDNA encodes a protein of 551 amino acid residues containing 12 membrane spanning domains, which shows 35 to $40 \%$ identity to the other OATs and OCTs.

From EST database search with hOAT4 amino acid sequence, BC021449 was found as a candidate for the OAT from mouse cDNA library. The isolated clone was identified as a mouse ortholog of human organic cation transporter-like 3 (ORCTL3). This clone has $36 \%$ to $40 \%$ and $35 \%$ to $37 \%$ identity in their amino acid sequences with mOATs and mOCTs, respectively. Phylogenetic analysis of the reported murine OATs, OCTs and human ORCTLs showed that mOCTL1 formed a cluster with OCT. Considering genomic location of OATs, OCTs and OCTL1 on the mouse genome, mOAT- $1,-3$ and -5 were located on the chromosome 19 except for mOAT-2 (on the chromosome 17). Human orthologs of OATs, except hOAT2 (on the chromosome 6), are all on the chromosome 11. In case of the mOCTs, three isoforms are located at different chromosomes (chromosome 1, 2, 12 and 17), but human orthologs of three isoforms of OCT are located on the chromosome 6 . Although human ORCTL3 (or OCTL1) and ORCTL4 (or OCTL2) are categorized as an OCT family, their genomic location is on the chromosome 3 , which is different from hOCTs. With respect to substrate
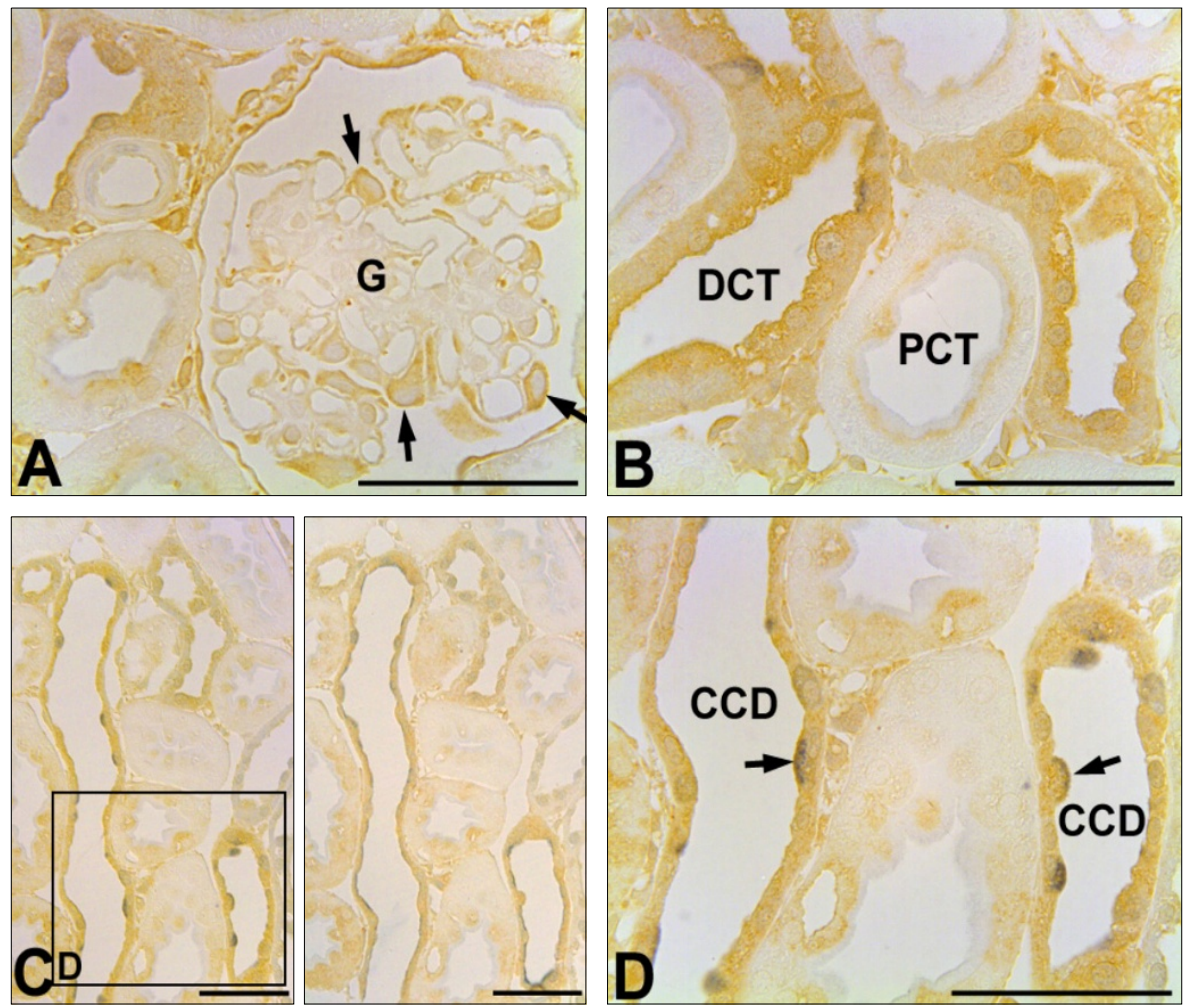

Figure 5. Immunohistochemical analysis of mOCTL1 in the kidney of mouse. Sections of mouse kidney in one-micrometer thick were incubated with polyclonal antibody against mOCTL1. Parietal epithelial cells and podocytes in glomerulus (A), apical membrane of proximal (B) and distal convoluted (C), and collecting tubule (D) was stained. C, Sections illustrating double immunostaining for mOCTL1 and $\mathrm{H}^{+}$-ATPase (left panel) or for mOCTL1 and AQP2 (right panel); $D$, Higher magnification of the area indicated by rectangle in $\mathrm{C}$. Arrows indicate $\mathrm{H}^{+}$-ATPase-positive intercalated cells in the cortical collecting duct (CCD). G, glomerulus; PCT, proximal convoluted tubule; DCT, distal convoluted tubule; CCD, cortical collecting duct (bar: 10 $\mu \mathrm{m})$. 
specificity, not only several OAT isoforms transport cationic substrate such as cimetidine but also OCT isoforms transport anionic substrate such as prostaglandin. Concerning OAT2, though it could transport organic anionic chemicals, the location on chromosome is different from those of other OAT isoforms (Kobayahi et al., 2002, 2005). For this reason, although it is designated as OCT, it causes controversy when one classifies the OAT2 and OCTL to member of OAT and OCT family, respectively.

In addition, OATs and OCTs have conserved motifs: 1) G-(X)3-D-R/K-X-G-R-R/K and D-R/K-XG-R; 2) E-(X)6-R; 3) P-E-S-P-R-X-L and P-E-T-K. It is to note that mOCTL1 also includes these motifs. The first conserved amino acid sequence of OAT including RST/URAT1 was $F$ in third amino acid from the start amino acid, $M$. The amino acid sequence of mOCTL1 was not matched with such rule (MAQF) like those of OCT. All amino acid sequences of human and murine OCT orthologs have conserved amino acid sequences in two sites but not OAT, IL (350 and 351 amino acids in mOCT1) and $\mathrm{Gl}$ (478 and 479 amino acids in mOCT1). These were also conserved in mOCTL1. On the other hand, with respect to hORCTL3, it was named only based on the rOCT1 amino acid homology without functional analysis. The EST revealed current study has $73 \%$ of amino acid homology with that of hORCTL3 (hOCTL1). Therefore we designated name of this clone as mOCTL1.

As shown in Figure 1, mOCTL1 has 12 putative membrane spanning domains, one extracellular long loop between the first and second transmembrane domains, and one intracellular long loop between the sixth and seventh transmembrane domains, which is all similar to the other OAT and OCT isoforms. In OATs and OCTs, multiple PKC phosphorylation sites were conserved in the intracellular long loop (Burckhardt and Wolff, 2000), but those of OCTL did not show such pattern. This result could indicate that the function of OCTL could be involved in different regulatory mechanism from that of OATs and OCTs.

The definition of OAT and OCT can be characterized by the substrate specificity. Unfortunately there was no report on uptake profile of OCTL. Furthermore, we could not identify the typical substrate(s) for mOCTL1 by using 11 isotope labeled compounds and employing the Xenopus laevis oocyte expression system $\left(\left[{ }^{14} \mathrm{C}\right]\right.$ para-aminohippurate, $\left[{ }^{3} \mathrm{H}\right]$ dehydroepiandrosterone sulfate, $\left[{ }^{3} \mathrm{H}\right]$ estrone sulfate, $\left[{ }^{14} \mathrm{C}\right]$ carnitine, $\left[{ }^{3} \mathrm{H}\right]$ prostaglandin $\mathrm{E} 2,\left[{ }^{3} \mathrm{H}\right]$ cyclo-adenosine monophosphate, $\left[{ }^{3} \mathrm{H}\right]$ estradiol $17 \beta$-D-glucuronide, $\left[{ }^{14} \mathrm{C}\right]$ tetraethyl ammonium bromide, $\left[{ }^{14} \mathrm{C}\right]$ uric acid, $\left[{ }^{3} \mathrm{H}\right]$ methotrexate and $\left[{ }^{3} \mathrm{H}\right]$ ochratoxin $\left.A\right)$. Even though $\left[{ }^{14} \mathrm{C}\right]$ salicylate was detected, a very small amount of uptake was seen and kinetic parameters were not able to evaluate. In our explanation, it may be difficult to act for mammalian functional protein in amphibian expression system. In RST (Mori et al., 1997), it was also called mouse organic cation transporter-like protein because it has amino acid identity to OCT1 without functional uptake experiment. Recently, RST was revealed as mouse ortholog of uric acid transporter (Hosoyamada et al., 2004) by functional uptake experiment. Therefore, it will be necessary further for functional test using various substrates in appropriate expression system, such as mammalian cell expression system, to clarify whether mOCTL1 belong to either OAT or OCT family.

For the tissue distribution of mOCTL1, the mRNA expression of mOCTL1 was observed in kidney. Almost all OAT and OCT isofoms are expressed in kidney except for mOAT6, which is predominantly expressed in olfactory mucosa and minimal expression in the testis. The tissue distribution of mOCTL1 is similar to that of RST (Mori et al., 1997) and rOAT5 (Anzai et al., 2005) where the expressions are kidney-specific. The tissue distribution of human ortholog of mOCTL1 using Northern blot analysis was detected ubiquitously in all tested organs (Nishiwaki et al., 1998). This discrepancy in tissue distribution among different species is unique in those of OATs and OCTs orthologs. On the Western blot analysis for mOCTL1, we detected the corresponding band (about $90 \mathrm{kDa}$ ). This band disappeared when the antibodies were absorbed with the synthesized oligopeptide antigen. The expected molecular weight, calculated from the amino acid contents, was $60.8 \mathrm{kDa}$. Our result showed that mOCTL1 contained $\mathrm{N}$-glycosylation sites and it would most likely affect the function of mOCTL1.

The localization of organic ion transporter in the intra-nephron is very important for understanding the direction of movement of the organic ionic substances. The OAT1 and OAT3 proteins are localized to the basolateral membrane, and OAT2 and OAT4 in the apical membrane of nephrons (Hosoyamada et al., 1999; Cha et al., 2001; Babu et al., 2002; Kojima et al., 2002). OCT1 and OCT2 proteins are localized in the basolateral membrane of renal tubules. The immunohistochemical results showed that mOCTL1 proteins are localized at the apical membrane of nephrons. Interestingly, mOCTL1 was identified in the podocytes and parietal epithelial cells in the glomerulus. In addition, mOCTL1 was detected in the apical site of 
proximal and distal convoluted tubule and diffused staining pattern was detected in connecting and collecting tubule. It is probable that mOCTL 1 may contribute to the transepithelial movement of drugs/chemicals at apical site of tubules and in the glomerulus.

In conclusion, we report in the present study on the identification of mOCTL1 that was uniquely expressed in mouse kidney. The amino acid homology and phylogenetic analyses showed that this protein may belong to either the organic anion or cation transporter family. It will be necessary to conduct extensive research on the transport properties of mOCTL1 for clear classification.

\section{Acknowledgement}

This work was supported by the Inha University Research Grant.

\section{References}

Anzai N, Jutabha $\mathrm{P}$, Enomoto A, Yokoyama $\mathrm{H}$, Nonoguchi $\mathrm{H}$, Hirata T, Shiraya K, He X, Cha SH, Takeda M, Miyazaki H, Sakata T, Tomita K, Igarashi T, Kanai Y, Endou H. Functional characterization of rat organic anion transporter 5 (Slc22a19) at the apical membrane of renal proximal tubules. J Pharmacol Exp Ther 2005;315:533-44

Babu E, Takeda M, Narikawa S, Kobayashi Y, Enomoto A, Tojo A, Cha SH, Sekine T, Sakthisekaran D, Endou H. Role of human organic anion transporter 4 in the transport of ochratoxin A. Biochim Biophys Acta 2002;1590:64-75

Burckhardt G, Wolff NA. Structure of renal organic anion and cation transporters. Am J Physiol Renal Physiol 2000;278: F853-66

Cha SH, Sekine T, Fukushima JI, Kanai Y, Kobayashi Y, Goya $\mathrm{T}$, Endou $\mathrm{H}$. Identification and characterization of human organic anion transporter 3 expressing predominantly in the kidney. Mol Pharmacol 2001;59:1277-86

Enomoto A, Kimura H, Chairoungdua A, Shigeta $Y$, Jutabha $\mathrm{P}$, Cha SH, Hosoyamada M, Takeda M, Sekine T, Igarashi T, Matsuo H, Kikuchi Y, Oda T, Ichida K, Hosoya T, Shimokata $\mathrm{K}$, Niwa T, Kanai $\mathrm{Y}$, Endou $\mathrm{H}$. Molecular identification of a renal urate anion exchanger that regulates blood urate levels. Nature 2002a;417:447-52

Enomoto A, Wempe MF, Tsuchida $H$, Shin $H J$, Cha A, Sakamoto A, Niwa T, Kanai Y, Anders MW, Endou H. Molecular identification of a novel carnitine transporter specific to human testis. Insights into the mechanism of carnitine recognition. J Biol Chem 2002b;277:36262-71

Eraly SA, Monte JC, Nigam SK. Novel slc22 transporter homologs in fly, worm, and human clarify the phylogeny of organic anion and cation transporters. Physiol Genomics 2004;18:12-24

Eun BK, Lee B, Kang HM. Cloning and expression of cryptochrome2 cDNA in the rat. Mol Cells 2001;12:286-91
Grundemann D, Gorboulev V, Gambaryan S, Veyhl M, Koepsell H. Drug excretion mediated by a new prototype of polyspecific transporter. Nature 1994;372:549-52

Hosoyamada M, Sekine T, Kanai Y, Endou H. Molecular cloning and functional expression of a multispecific organic anion transporter from human kidney. Am J Physiol 1999; 276:F122-8

Hosoyamada M, Ichida K, Enomoto A, Hosoya T, Endou H. Function and localization of urate transporter 1 in mouse kidney. J Am Soc Nephrol 2004;15:261-8

Jung SY, Kwak JO, Kim HW, Kim DS, Ryu SD, Ko CB, Cha $\mathrm{SH}$. Calcium sensing receptor forms complex with and is up-regulated by caveolin-1 in cultured human osteosarcoma (Saos-2) cells. Exp Mol Med 2005;37:91-100

Kekuda R, Wang H, Huang W, Pajor AM, Leibach FH, Devoe LD, Prasad PD, Ganapathy V. Primary structure and functional characteristics of a mammalian sodium-coupled high affinity dicarboxylate transporter. J Biol Chem 1999;274: 3422-9

Kobayashi Y, Ohshiro N, Shibusawa A, Sasaki T, Tokuyama $\mathrm{S}$, Sekine T, Endou H, Yamamoto T. Isolation, characterization and differential gene expression of multispecific organic anion transporter 2 in mice. Mol Pharmacol 2002;62:7-14

Kobayashi Y, Ohshiro N, Sakai R, Ohbayashi M, Kohyama $\mathrm{N}$, Yamamoto T. Transport mechanism and substrate specificity of human organic anion transporter 2 (hOAT2 [SLC22A7]. J Pharm Pharmacol 2005;57:573-8

Koepsell H. Organic cation transporters in intestine, kidney, liver, and brain. Annu Rev Physiol 1998;60:243-66

Kojima R, Sekine T, Kawachi M, Cha SH, Suzuki Y, Endou $\mathrm{H}$. Immunolocalization of multispecific organic anion transporters, OAT1, OAT2, and OAT3, in rat kidney. J Am Soc Nephrol 2002;13:848-57

Kusuhara H, Sekine T, Utsunomiya-Tate N, Tsuda M, Kojima $\mathrm{R}$, Cha SH, Sugiyama Y, Kanai Y, Endou H. Molecular cloning and characterization of a new multispecific organic anion transporter from rat brain. J Biol Chem 1999;274:13675-80

Kwak JO, Kim HW, Oh KW, Kim DS, Han KO, Cha SH. Co-localization and interaction of organic anion transporter 1 with caveolin-2 in rat kidney. Exp Mol Med 2005;37:204-12

Monte JC, Nagle MA, Eraly SA, Nigam SK. Identification of a novel murine organic anion transporter family member, OAT6, expressed in olfactory mucosa. Biochem Biophys Res Commun 2004;323:429-36

Mori K, Ogawa Y, Ebihara K, Aoki T, Tamura N, Sugawara A, Kuwahara T, Ozaki S, Mukoyama M, Tashiro K, Tanaka I, Nakao K. Kidney-specific expression of a novel mouse organic cation transporter-like protein. FEBS Lett 1997;417: 371-4

Nishiwaki T, Daigo Y, Tamari M, Fujii Y, Nakamura Y. Molecular cloning, mapping, and characterization of two novel human genes, ORCTL3 and ORCTL4, bearing homology to organic-cation transporters. Cytogenet Cell Genet 1998;83:251-5

Okuda M, Saito H, Urakami Y, Takano M, Inui K. cDNA cloning 
and functional expression of a novel rat kidney organic cation transporter, OCT2. Biochem Biophys Res Commun 1996; 224:500-7

Pritchard JB, Miller DS. Proximal tubular transport of organic anions and cations. In: The Kidney: Physiology and Pathology, edited by Seldin DW and Giebisch G. New York: Raven, 1992, 2921-45

Roch-Ramel F, Diezi J. Renal transport of organic ions and uric acid. In: Diseases of the Kidney, edited by Schrier RW and Gottschalk CW, Boston, Ma: Little, Brown, 1997, 231-49

Roch-Ramel F, Besseghir K, Murer H. Renal excretion and tubular transport of organic anions and cations. In: Handbook of Physiology. Renal Physiology. Bethesda, MD: Am. Physiol Soc., 1992, sect.8, vol. II, chapt. 48, p.2189-262

Sekine T, Cha SH, Endou H. The multispecific organic anion transporter (OAT) family. Eur J Physiol 2000;440:337-50

Tamai I, Yabuuchi H, Nezu J, Sai Y, Oku A, Shimane M, Tsuji A. Cloning and characterization of a novel human $\mathrm{pH}$ - dependent organic cation transporter, OCTN1. FEBS Lett 1997;419:107-11

Ullrich KJ. Specificity of transporters for "organic anions" and "organic cations" in the kidney. Biochim Biophys Acta 1994;1197:45-62

Ullrich KJ. Renal transporters for organic anions and organic cations. Structural requirements for substrates. J Membr Biol 1997; 158:95-107

Wu X, Prasad PD, Leibach FH, Ganapathy V. cDNA sequence, transport function, and genomic organization of human OCTN2, a new member of the organic cation transporter family. Biochem Biophys Res Commun 1998; 246:589-95

Youngblood GL, Sweet DH. Identification and functional assessment of the novel murine organic anion transporter Oat5 (Slc22a19) expressed in kidney. Am J Physiol 2004; 287:F236-44 